\title{
UN ANÁLISIS PROVINCIAL COMPARATIVO \\ DE LA LOCALIZACIÓN DE ACTIVIDADES \\ PROFESIONALES EN ÁREAS RURALES ${ }^{1}$
}

Inmaculada Polo Angel Paniagua*

Los Servicios a Empresas están considerados como la nueva fuente de empleo por la gran flexibilidad que presentan. Estos servicios cubren actividades demandadas desde las muy cualificadas hasta las menos, consiguiendo servicios de gran calidad con gran flexibilidad en la producción y por tanto en el empleo, variables predominantes en el entorno económico actual.

El crecimiento de estos servicios se ve potenciado por las distintas actividades laborales nuevas que van surgiendo, orientadas principalmente hacia un trabajo autónomo y distintos proyectos individuales.

Tanto el trabajo autónomo como el teletrabajo es una de las soluciones que se están buscando en los países europeos para intentar reducir los altos índices de desempleo, enfocándolos principalmente a la incorporación al mercado laboral de la mujer, siendo el que más aceptación tiene el trabajo a tiempo parcial (Rubalcaba, 2001).

Las distintas oportunidades de empleo varían según el tipo de país con diferencias notables en relación a las condiciones del mercado laboral, factores culturales y trabajo autónomo.

Como han indicado Hoggart y Paniagua (2001), durante el período 19951999 el crecimiento total del número de empresas en España subió hasta el 9,5\% en lugares con menos de 10.000 habitantes comparado con el 8,7\% de los grandes núcleos. No hay una gran diferencia aquí, pero para compañías de los sectores profesional y servicios las cifras fueron $41,3 \%$ y $27,3 \%$.

\footnotetext{
* Investigadores del CSIC.
} 
Aun así, uno tiene que ser cuidadoso interpretando este panorama de forma tan positiva. Los núcleos con menos de 10.000 habitantes en Cataluña sumaban el $21,1 \%$ de todas las empresas de la región en 1999 , pero solamente el $14,4 \%$ de las consideradas más profesionales y de servicios.

El panorama en zonas agrícolas y de interior es menos positivo. Así, en Castilla La Mancha los porcentajes para lugares con menos de 10.000 habitantes en 1999 eran $51,7 \%$ (todas las empresas) y $31,8 \%$ (empresas de servicios y profesionales), con el 19,4\% y 10,6\% en Andalucía (Hoggart y Paniagua, 2001).

El propósito de este trabajo es profundizar en las tendencias apuntadas a un nivel provincial y municipal, en escenarios espaciales contrastados, con el fin de explorar en detalle en núcleos rurales el comportamiento de las empresas de servicios profesionales y financieros.

\section{METODOLOGÍA Y FUENTES DE ESTUDIO}

Estudio realizado de las provincias de Ciudad Real, Girona y Guadalajara, a partir de los datos facilitados por la Cámara de Comercio e Industria del año 2001 en municipios de población de hecho de menos de 5.000 habitantes. Estas provincias han sido elegidas por los contrastes que ofrecen entre una provincia dinámica de litoral y otras provincias del interior para analizar la incidencia que tienen las empresas de servicios en el mundo rural, por las diferencias regionales y locales, para extrapolar a cada ámbito territorial (Velasco, 1998).

Se han incorporado datos del Instituto Nacional de Estadística relacionados con el código provincial y la población de hecho, y del Impuesto de Actividades Económicas (IAE) del año 95-99. Estos datos permiten un análisis longitudinal entre los distintos ámbitos territoriales, en función de la localización de los profesionales de servicios a empresas, segmentando el estudio por tramos de población y por actividad principal.

\section{EVOLUCIÓN DE LAS EMPRESAS DE SERVICIOS PROFESIONALES}

Debido al gran dinamismo y la evolución de las pequeñas empresas en los núcleos rurales de menos de 5.000 habitantes se aprecia un notable incremento de las empresas de servicios en todas las áreas estudiadas en los años citados.

Así, por ejemplo en Girona, los servicios jurídicos (841) experimentan un crecimiento espectacular en los tramos de población de 500-1000 habitantes en el año 99 , con un crecimiento sostenido en los demás tramos de población, cosa que no ocurre en poblaciones de más de 10.000 habitantes que decrecen.

En las actividades (842), los servicios financieros y contables aumentan principalmente en los tramos de 0-500 habitantes y en los tramos comprendidos entre 500-1000 habitantes. No ocurre lo mismo en los siguientes tramos de 
población donde se mantiene el crecimiento o decrece en poblaciones de más de 10.000 habitantes.

En relación con los datos del año 95, en el año 99 los servicios técnicos (ingeniería, arquitectura, urbanismo, etc.) 843, mantienen un crecimiento en los tramos de población de menos de 5.000 habitantes y decrecen en los tramos de más de 5.000 habitantes.

Pero es en los servicios de publicidad, relaciones públicas y similares, código 844, donde podemos observar un crecimiento más espectacular en general en todos los núcleos independientemente de su población con un $46 \%$ del total sobre el año 95.

En las actividades correspondientes con el código 846-847, empresas de estudios de mercado y servicios integrales, el crecimiento se mantiene moderado en el año 99. No ocurre lo mismo con las actividades referidas a otros servicios prestados a empresas, código 849 , donde se incrementan en un $54,4 \%$ en el año 99 respecto al año 95, en todos los tramos de población.

En provincias como Ciudad Real donde por su situación geográfica y debido al mayor impulso que han tomado todas las comunicaciones se produce un mayor crecimiento en los servicios a empresas en el año 99 en relación al año 95.

Así, por ejemplo, las empresas de servicios jurídicos (841), aumentan principalmente en el año 99 en los tramos de población de menos de 5.000 habitantes disminuyendo en los tramos superiores.Ocurre lo mismo en las empresas de servicios financieros y contables (842), donde el incremento es mayor en los núcleos rurales inferiores a 5.000 habitantes.

Tanto las empresas de servicios técnicos y de ingeniería (843), como las empresas de servicios de publicidad, relaciones públicas y similares (844) tienen en los tramos inferiores a 5.000 habitantes su incremento mayor en el año 99, con relación a los datos del año 95, que en núcleos superiores a 5.000 habitantes se mantienen o bien tienen un crecimiento sostenido.

En Guadalajara, los servicios a empresas profesionales apenas sufren variación en los datos contrastados del año 95 y 99, se mantienen en ambas fechas en los tramos estudiados en núcleos rurales con población inferior a 5.000, donde se aprecia un ligero incremento es en los tramos superiores a esta población.

\section{PRINCIPALES CARACTERÍSTICAS DE LAS EMPRESAS DE SERVICIOS PROFESIONALES POR PROVINCIAS}

Actividad Principal

Las actividades principales en el estudio realizado en las tres provincias contrastadas son las que pertenecen a los grupos 83 , auxiliares financieros y de seguros, y actividades inmobiliarias que representan un $36,2 \%$ del total de los casos 
analizados. Por último, el grupo que comprende los servicios prestados a empresas y organismos (84) representa el 19\% del total estudiado.

Dentro del grupo 83, la actividad principal predominante en los núcleos de población estudiados es la relacionada con la promoción inmobiliaria de edificaciones, la compraventa en nombre y por cuenta propia, construidas directamente con el fin de venderlas (8332 código de actividad principal); con un porcentaje del $26,8 \%$ sobre las demás actividades principales y a su vez los servicios prestados a empresas como servicios jurídicos y servicios financieros y contables, del grupo 84 representando las categorías principales (842) servicios financieros y contables el $6,1 \%$ del total, y los servicios de publicidad relaciones públicas y similares, código 844 .

Actividad Secundaria

En el estudio por provincias de la dedicación secundaria es igualmente significativo el alto porcentaje que existe del código 8332 anteriormente mencionado, relacionado con actividades principales diversas, como por ejemplo comercio, construcciones, transporte, etc.

Otras actividades secundarias importantes son las relacionadas con los servicios prestados a empresas grupo (84), relacionados directamente con actividades principales que no forman parte necesariamente de este grupo como intermediarios del comercio, industrias cárnicas, restaurantes con dedicaciones secundarias relacionadas con empresas de estudios de mercado, servicios financieros y contables, servicios administrativos y otros servicios equivalentes profesionales para empresas.

\section{Formas Jurídicas}

Dentro de las formas jurídicas, la principal en la mayoría de los tramos de población es la Sociedad Limitada formada entre 1 y 50 socios con un capital mínimo de 500.000 pesetas, con régimen autónomo para socios trabajadores $(62,2 \%)$. Seguida con un amplio margen de diferencia sobre la Sociedad Anónima, con un $15,2 \%$ de los casos estudiados, formada con un mínimo de 3 socios con un capital aportado mínimo de 10.000 .000 y con régimen autónomo para socios trabajadores (Velasco, 1998). Otra forma jurídica es la del empresario autónomo que corresponde a un $12 \%$ de los datos contrastados en todas las provincias.

Tamaño de la Empresas

En la mayoría de las empresas donde aparece el dato del número de empleados existe en amplio margen de diferencia en los campos de empleados de 1 a 5 , existiendo única y exclusivamente dos empresas que superen la cifra de más de 
500 empleados, tratándose en la mayoría de los casos de microempresas, pequeñas empresas con distintas actividades, desde las más tradicionales como por ejemplo el comercio artesanal, hasta las más cualificadas en alta tecnología de servicios técnicos, etc. Estas empresas siguen un modelo organizativo extenso, desde las cooperativas hasta el tipo de empresa familiar más tradicional (Velasco, 1998).

\section{LOCALIZACIÓN DE LOS SERVICIOS PROFESIONALES A EMPRESAS}

Debido a los procesos de internacionalización se producen a la vez movimientos de concentración. "En la medida en que Europa esté cada vez más integrada y que las empresas se beneficien de dicha integración, Europa podrá considerarse como una única región en la que operan empresas a nivel internacional" (Rubalcaba, 2001). Esto repercute en los servicios a empresas que desde siempre se habían considerado de ámbito local, y se está produciendo el proceso de internacionalización debido al cáracter multinacional de los clientes.

Los servicios a empresas tienden a concentrarse en municipios de mayor renta y cerca de organismo oficiales, ministerios, administraciones regionales etc., aunque en la actualidad se está siguiendo la pauta de la descentralización debido a la gran flexibilidad que presentan estos servicios que permiten la no confluencia en el mismo área de localización de la demanda y la oferta.

Los factores de la localización pueden ser agrupados en factores de demanda, factores de oferta y factores de mercado, como señala Rubalcaba (2001).

Así como los factores tradicionales del coste dejan de tener importancia relativa frente a la proximidad y cercanía del cliente.

Los profesionales de los servicios de empresas se encuentran situados en torno a las capitales de provincias y municipios punteros y próximos a las vías de comunicación principales, con la excepción de Girona donde se encuentran dispersos por las distintas comarcas y siguiendo el litoral por el determinado carácter turístico de las zonas.

\section{LOCALIZACIÓN: UN ANÁLISIS COMPARATIVO DE LOS AMBITOS PROVINCIALES SELECCIONADOS}

\section{Girona}

La localización de las empresas en la provincia de Girona viene determinada por su situación geográfica entre el mar y la montaña y las distintas comarcas de la provincia, así por ejemplo en la zona el Baix Empordá, se concentran en la franja del litoral, siendo las principales empresas las dedicadas a la construcción y promociones de edificaciones y de terrenos, ocurre lo mismo con la zona de la comarca de la Selva con municipios punteros como Blanes, Lloret del Mar, etc., y 
municipios como Riudellos de la Selva, o Sta. Coloma de Farnés donde la localización gira entorno al eje de comunicaciones que es la Nacional II , y sobre todo por su proximidad con la capital Girona, es en estos municipios donde las empresas de servicios tienen un mayor porcentaje, unidas con las empresas de construcción y de dedicación turística.

Otro área de fuerte concentración es la comarca de La Cerdanya que por su situación geográfica como paso fronterizo natural entre Cataluña, Francia y el Principado de Andorra y con una actividad económica concentrada fundamentalmente en el turismo y deportes de ocio atrae a un gran número de empresas de servicios, situándose principalmente en Puigcerdá, capital de la comarca, y en el entorno de las estaciones de esquí de la Molina, Alp, etc., donde podemos encontrar empresas no solamente dedicadas al turismo tanto en construcción como en alquileres, como empresas de servicios financieros y contables de gestión administrativa, servicios jurídicos, servicios técnicos y actividades varias como industrias de piedra natural que como actividad secundaria tienen la promoción de edificaciones, y bares y cafés con actividad secundaria de alquiler de edificaciones.

Resulta altamente significativo como en núcleos de población de hasta 500 habitantes se encuentran localizadas empresas de construcción de edificaciones (código 501) que a su vez como dedicación secundaria tienen la actividad 833 de promoción inmobiliaria hecho igualmente extrapolable a otros tramos de población superiores a 500 habitantes.

Actividad a destacar por su concentración de empresas tanto en tramos de población más pequeña como en núcleos de población mayor es la relacionada con la actividad $6 . .$. servicios de restaurantes, con mayor volumen de empresas en la actividad 673 (cafés bares, con o sin comida).

Pero el mayor volumen de empresas dentro de la provincia de Girona se encuentra en la actividad $8 . .$. , instituciones financieras, promociones inmobiliarias, servicios financieros y contables, servicios técnicos y multiservicios a distintas empresas, como traducción, publicidad, marketing etc., lo que supone un $55 \%$ de los casos analizados.

Principalmente son empresas de un tamaño medio o microempresas, tipo empresa familiar (Velasco, 1998), con un máximo de hasta 25 empleados.

\section{Ciudad Real}

Por el dinamismo actual que está tomando la comunidad de Castilla-La Mancha, con relación a su situación geográfica que determina una evolución positiva por los bajos costes de instalación, junto con las mejoras de accesibilidad en las infraestructuras de transporte y telecomunicaciones, se están desarrollando unas ciudades medias, cabeceras comarcales y sistemas locales dinámicos con un mínimo de demanda para concentrar servicios empresariales. 
Existe predominio de la llamada economía social (empresas familiares, Sociedad Limitada, Sociedad Anónima Laboral, cooperativas...) con un reforzamiento de la pequeña empresa monoplanta protagonista del relanzamiento. (Alonso y Méndez, 2000).

Ciudad Real, es la provincia dentro de la Comunidad de Castilla-La Mancha que mayor impulso ha tomado en las empresas de servicios. Las áreas más activas coinciden con pequeñas ciudades o con núcleos rurales dispersos por su territorio, dado que las mejoras en las comunicaciones aproximan a las empresas a los mercados circundantes, principalmente Madrid, dan un mayor dinamismo a las periferias emergentes. (Alonso y Méndez, 2000).

Dentro de la provincia de Ciudad Real, la localización de las empresas de servicios viene marcada por la distribución de la población y por el impulso que han tomado las nuevas infraestructuras en las comunicaciones; dado que la distancia con Madrid se ha reducido notablemente gracias a la línea de Alta Velocidad, que sitúa a Ciudad Real a menos de 50 minutos de la capital y próxima a otros centros urbanos del Levante y del sur de España. Este factor es determinante a la hora de localizar dichas empresas, situándose estas principalmente entorno a los ejes de comunicación, así por ejemplo están localizadas en la órbita de Ciudad Real capital, eje de la N-IV, y la N-420 que une la provincia con Madrid por carretera.

El tren de Alta Velocidad (AVE) ha impulsado positivamente el crecimiento de Ciudad Real capital, con una gran diversificación de los servicios empresariales: ferias, exposiciones y congresos que se vienen realizando en la ciudad, que a su vez repercute en la localización de las empresas de servicios. Para la población estos datos son un reclamo importante, con un crecimiento demográfico bastante alto, lo que determina una expansión a su vez urbanística, con un considerable aumento de las empresas con actividad principal de promociones inmobiliarias (833) ubicadas en municipios de menos de 5.000 habitantes, estas empresas representan un $19 \%$ del total de las empresas de servicios.

Ocurre lo mismo en tramos de población de menos de 1.000 habitantes donde la actividad principal es la relacionada con las edificaciones y las construcciones siendo predominante la actividad 833, conjuntamente con las actividades relacionadas con los servicios técnicos de urbanismo y arquitectura (843); siendo significativo la localización de dos de estas empresas en núcleo de población de 600 habitantes próximo a Ciudad Real (Poblete).

Otros municipios con un alto índice de empresas de servicios son: Calzada de Calatrava, Chillón, Torrenueva, Herencia, etc.

Guadalajara

Por su privilegiada situación, localizada en el centro de la península, y por el carácter de la red viaria española, la provincia de Guadalajara dispone de una 
amplia red de accesos a su territorio y de vías de comunicación interiores a través de cualquier medio de transporte, lo que determina la localización de las empresas de servicios. Factores claves son la proximidad a ciudades relevantes como es la capital Guadalajara o a ejes importantes como es la autovía de Aragón, que enlaza Madrid con Zaragoza, Barcelona y Francia.

Como en el resto del estudio de las distintas provincias existe una gran concentración de empresas cuyo código de actividad corresponde al 833, es decir promociones inmobiliarias en todos los núcleos urbanos sin distinción de tramos de población, que representa un $18,7 \%$ sobre el resto de las actividades. Concentrándose principalmente estas empresas en el entorno de Guadalajara, próximas al embalse de Entrepeñas y cerca de los ejes de comunicación principales (N-211).

Los principales núcleos de población donde podemos encontrar este tipo de empresas de servicios son: Brihuega, Pastrana, Sacedón, Molina y en el entorno de Guadalajara capital.

Dentro de la provincia de Guadalajara cabe destacar en municipios de menos de 500 habitantes empresas con actividades muy diversas desde empresas de construcción, código 501, con dedicación secundaria de promoción de edificaciones (833) hasta empresas de transporte de mercancías por carretera (código 722), y las empresas relacionadas con los servicios inmobiliarios y con los servicios prestados a empresas. Como ocurre en los municipios de Caspueñas, Durón, Uceda, El Olivar, etc., donde encontramos empresas de servicios técnicos, financieros y contables.

\section{CONCLUSIÓN}

La transformación del mundo rural en las provincias estudiadas nos demuestra como las pautas de comportamiento en las localizaciones de las empresas de servicios profesionales van cambiando a lo largo de los años, produciéndose un crecimiento de las empresas en las áreas rurales de menos de 5.000 habitantes.

Por el desarrollo endógeno y sostenido de las zonas rurales a través de la diversificación de sus economías y para evitar un mayor exódo rural se están produciendo cambios considerables en las políticas comunitarias y regionales para fomentar el cambio estructural en el mundo rural, con mayor implantación de las nuevas economías y empresas profesionales.

Así, por ejemplo, con los datos que manejamos del año 95 y 99 podemos apreciar como en la provincia de Girona en el año 99 aumentan considerablemente las empresas de servicios profesionales, en núcleos menores de 5.000 habitantes distribuidos por todo el litoral, en función de sus actividades económicas orientadas principalmente al turismo; como son las empresas dedicadas a la construcción y promociones de edificaciones y de terrenos, siendo la comarca de la Selva, junto con la zona del Baix Empordá, los principales focos de concentración de estas empresas, ya en el interior de la provincia su localización está dispuesta en torno a 
las grandes cabeceras comarcales como ocurre en la comarca de la Cerdanya, donde los núcleos principales de localización son en el area de Puigcerdá, Llívia y Alp, donde su economía actual se basa en un sector terciario enfocado principalmente a un turismo invernal vínculado principalmente al comercio y a la oferta de esquí, junto con las actividades tradicionales de sectores como la alimentación etc. Se encuentran casos donde empresas con dedicación principal pertenecientes al grupo 6, comercios restaurantes y hospedajes, que como dedicación secundaria tienen actividades relacionadas con los servicios de gestión administrativa, multiservicios intensivos en personal, etc.

Es en la Comunidad de Castilla-La Mancha, donde por el impulso de las comunicaciones y las nuevas estratégias para promover un desarrollo local y regional, se aprecia una mayor localización de las empresas de servicios profesionales en los ejes de las cabeceras comarcales, dando gran relevancia a las periferias emergentes. (Alonso y Méndez, 2000).

Como es el caso de la provincia de Ciudad Real, con núcleos rurales satélites en torno a las grandes ciudades y ejes de comunicación, focos de atracción principal para las localizaciones de las empresas de servicios profesionales.

En Guadalajara, la localización de empresas de servicios viene determinada por las líneas principales de comunicación, tanto férreas como por carretera, dada la atracción central de Madrid, que se organizan de manera radial cruzando toda la región. Este hecho es importante en el proceso de descongestión económico-industrial de la capital, que privilegió a determinadas zonas de Guadalajara, con la prologación natural del Corredor del Henares. 
NOTAS

${ }^{1}$ Este texto forma parte del proyecto PB98-644 financiado por el Programa de Promoción General del Conocimiento del Ministerio de Ciencia y Tecnología.

\section{BIBLIOGRAFÍA}

HOGGART, K. y PANIAGUA, A. (2001): “The restructuring a rural Spain?", en Journal of Rural Studies, 17, pp. 69-70.

MENDEZ, R., RODRIGUEZ, J. y MECHA, R. (2000): “Medios de innovación y desarrollo en sistemas productivos locales de Castilla-La Mancha”, en ALONSO, J.L. y MENDEZ ,R., (Eds.), Innovación, pequeña empresa y desarrollo local en España, Civitas, Madrid, pp. 101-123.

RUBALCABA, L., (2001): La economía de los servicios a empresas en Europa, Pirámide, Madrid, pp. 83-120 y 401-433.

VELASCO, R., (1998): La creación de empresas en España, Círculo de Empresarios Vascos, Bilbao, pp. 50-62 
CUADRO No 1: Análisis comparativo de distribución de empresas de servicios por provincias y tramos de población. Año 2001.

\begin{tabular}{|c|c|c|c|c|c|c|c|c|c|c|c|c|}
\hline \multirow{2}{*}{$\begin{array}{l}\text { GIRONA } \\
0-500\end{array}$} & 819 & 831 & 832 & 833 & 834 & 841 & 842 & 843 & 844 & 845 & 846 & 849 \\
\hline & & & 4 & 58 & 13 & & 12 & 20 & 8 & 2 & 3 & 29 \\
\hline $500-1000$ & 2 & & 5 & 56 & 12 & 1 & 2 & 15 & 5 & 8 & 6 & 13 \\
\hline $1000-2000$ & 1 & & 10 & 97 & 34 & 1 & 13 & 18 & 10 & 9 & 3 & 19 \\
\hline $2000-5000$ & 4 & 3 & 35 & 255 & 196 & 1 & 52 & 40 & 39 & 22 & 10 & 130 \\
\hline $5000-10000$ & 5 & & 25 & 152 & 83 & 5 & 29 & 28 & 19 & 8 & 5 & 58 \\
\hline MÁS DE 10000 & 2 & & 19 & 98 & 55 & 3 & 22 & 15 & 13 & 5 & 7 & 33 \\
\hline
\end{tabular}

\begin{tabular}{|l|c|c|c|}
\hline GUADALAJARA & 832 & 833 & 834 \\
\cline { 2 - 4 } $0-500$ & & 7 & 1 \\
\hline $500-1000$ & & 2 & \\
\hline $1000-2000$ & & 9 & 1 \\
\hline $2000-5000$ & 3 & 8 & \\
\hline MÁS DE 10000 & & 1 & \\
\hline
\end{tabular}

\begin{tabular}{|c|c|c|c|c|c|}
\hline 842 & 843 & 844 & 845 & 846 & 849 \\
\hline & 4 & 2 & & & 3 \\
\hline & 1 & & & & \\
\hline 7 & 5 & 4 & 1 & 1 & 6 \\
\hline 2 & 2 & & 1 & & 5 \\
\hline & & 1 & & & 1 \\
\hline
\end{tabular}

\begin{tabular}{|l|c|c|}
\hline CIUDAD REAL & 832 & 833 \\
\cline { 2 - 3 } $500-1000$ & & \\
\hline $1000-2000$ & & 3 \\
\hline $2000-5000$ & 1 & 7 \\
\hline $5000-10000$ & 11 & 14 \\
\hline MÁS DE 10000 & 6 & 1 \\
\hline
\end{tabular}

\begin{tabular}{|c|c|c|c|}
\hline 841 & 842 & 843 & 844 \\
\hline & & 2 & \\
\hline 1 & & & \\
\hline & 7 & 3 & \\
\hline 1 & 5 & 4 & 1 \\
\hline & 4 & & 1 \\
\hline
\end{tabular}

\begin{tabular}{|l|}
\hline 849 \\
\hline \\
\hline 5 \\
\hline 5 \\
\hline 5 \\
\hline 4 \\
\hline
\end{tabular}

FUENTE: Elaboración propia, datos de la Cámara de comercio e industria. Año 2001.

819: otras instituciones financieras.

831: auxiliares financieros.

832: auxiliares de seguros.

833: promoción inmobiliaria. 
834: servicios relativos a la propiedad inmobiliaria y a la propiedad industrial.

841: servicios jurídicos.

842: servicios financieros y contables.

843: servicios técnicos.

844: servicios de publicidad, relaciones públicas y similares.

845: explotación electrónica por cuenta de terceros.

846: empresas de estudio de mercado.

849: otros servicios prestados a empresas. 